\title{
A First-in-Human Study of ${ }^{68}$ Ga-Nanocolloid PET/CT Sentinel Lymph Node Imaging in Prostate Cancer Demonstrates Aberrant Lymphatic Drainage Pathways
}

\author{
Jacki A. Doughton ${ }^{1,2}$, Michael S. Hofman ${ }^{1,2}$, Peter Eu ${ }^{1}$, Rodney J. Hicks ${ }^{1,2}$, and Scott Williams ${ }^{1,2}$ \\ ${ }^{I}$ Department of Radiation Oncology and Cancer Imaging, Peter MacCallum Cancer Centre, Melbourne, Victoria, Australia; and \\ ${ }^{2}$ University of Melbourne, Melbourne, Victoria, Australia
}

\begin{abstract}
Our goal was to assess the feasibility, safety, and utility of a novel ${ }^{68} \mathrm{Ga}-$ nanocolloid radiotracer with PET/CT lymphoscintigraphy for identification of sentinel lymph nodes (SLNs). Methods: This was a pilot study of patients from a tertiary cancer hospital who required insertion of gold fiducials for prostate cancer radiation therapy. Participation did not affect cancer management. Ultrasound-guided transperineal intraprostatic injection of a PET tracer (iron oxide nanocolloid labeled with ${ }^{68} \mathrm{Ga}$ ) was performed after placement of the fiducials. PET/CT lymphoscintigraphy imaging took place at approximately 45 and 100 min after injection of the tracer. The study was monitored using a Bayesian design with the assumption that at least 1 SLN could be identified in at least two thirds of cases with more than $80 \%$ confidence. Results: SLN identification was successful in all 5 participants, allowing completion of the pilot study as per protocol. No adverse effects were observed. Unexpected potential pathways for transit of malignant cells, as well as the expected regional drainage pathways, were discovered. Rapid tracer drainage to pelvic bone, perivesicular, mesorectal, inguinal, and Virchow nodes was identified. Conclusion: SLN identification using ${ }^{68} \mathrm{Ga}$-nanocolloid PET/CT can be successfully performed. Nontraditional pathways of disease spread were identified, including drainage to pelvic bone and to perivesicular, mesorectal, inguinal, and Virchow nodes. The prevalence of both aberrant and nonlymphatic pathways of spread should be further investigated with this technique.
\end{abstract}

Key Words: lymphoscintigraphy; lymph nodes; positron emission tomography; prostate neoplasms; sentinel lymph node; magnetite nanoparticles

J Nucl Med 2018; 59:1837-1842

DOI: 10.2967/jnumed.118.209171

$\mathbf{O}$ ur review of prostate cancer cases diagnosed in 2010 from the U.S. cancer registry of the National Cancer Institute showed that more men were initially diagnosed at stage N0 M1 disease (1,247 men) than at stage N1 M1 disease (558 men) (676 men were initially diagnosed with stage Nx M1 disease) $(1,2)$. Why does prostate cancer seem to spread distantly despite the apparent lack of involvement by regional lymph nodes? Possibilities

Received Feb. 3, 2018; revision accepted Apr. 30, 2018.

For correspondence or reprints contact: Jacki A. Doughton, Peter MacCallum Cancer Centre, 305 Grattan St., Melbourne, VIC 3000, Australia. E-mail: jacki.doughton@petermac.org

Published online May 4, 2018.

COPYRIGHT (c) 2018 by the Society of Nuclear Medicine and Molecular Imaging. include lymphatic drainage to nodes not routinely sampled at surgery or direct hematogenous spread, possibly by lymphaticovenous channels. Indeed, aberrant sites, defined as those not traditionally targeted by elective surgery or radiation (paraaortic, presacral, inguinal, and near the ventral abdominal wall), have been identified in up to a third of patients using sentinel lymph node (SLN) imaging with ${ }^{99 \mathrm{~m}} \mathrm{Tc}$-radiocolloid SPECT or SPECT/ CT (3). SLN imaging identifies the hypothetical first lymph node that is reached by metastasizing cancer cells (4). The known predisposition of metastasis to bone (5), in particular the pelvic and lower vertebral bones (6), supports the possibility of an alternative hematogenous route.

Despite the promise of SLN imaging, SPECT has technical limitations compared with PET/CT (7). We postulated that intraprostatic administration of ${ }^{68} \mathrm{Ga}$-nanocolloid combined with PET/ CT would leverage the technical advantages of PET/CT to provide sensitive identification of lymphatic pathways in clinically localized prostate cancer. Herein, we report a first-in-human (to our knowledge) trial assessing the feasibility, safety, and utility of PET/CT SLN imaging.

\section{MATERIALS AND METHODS}

\section{Study Population}

Patients who required implantation of gold fiducial markers into the prostate before definitive prostate cancer radiation therapy were prospectively recruited into our institutional review board-approved clinical study (PMCC HREC project 13/80, National Ethics Application Form submission code AU/1/1903112). All subjects gave written informed consent.

Inclusion criteria consisted of biopsy-proven prostate adenocarcinoma; the availability of serum prostate-specific antigen level and routine staging scan (CT of abdomen/pelvis and bone scan) results; a clinical stage of T1c-3b N0 M0; planned insertion of gold seed fiducial markers for image-guided radiation therapy; and fitness for general anesthetic. Patients could be receiving androgen deprivation or other systemic treatment at the time of radiation planning.

Patients were excluded if they had hip or other prostheses that might affect interpretation of PET/CT images (at physician discretion); previous abdominal or pelvic surgery, including inguinal hernia repair and surgery for undescended testis, or any previous surgery that could potentially interfere with abdominopelvic lymphatic drainage.

\section{Technique for SPECT/CT Lymphoscintigraphy}

To familiarize all staff with the study procedures in preparation for the experimental PET/CT tracer injection, the initial 3 participants were imaged with SPECT/CT lymphoscintigraphy using a technique 
for intraprostatic injection adapted from one previously described in the literature (8-14).

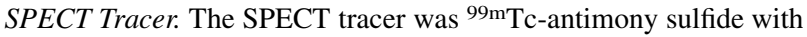
a size of $20 \pm 5 \mathrm{~nm}$. This size was similar to that of the ${ }^{68} \mathrm{Ga}$-iron oxide, which was also $20 \mathrm{~nm}$ with a hydrodynamic diameter of approximately $25 \mathrm{~nm}$.

SPECT/CT Injection Technique. Under general anesthesia, eight 20$\mathrm{MBq}$ doses of ${ }^{99 \mathrm{~m}} \mathrm{Tc}$-antimony sulfide colloid were injected into the prostate using a standard transperineal template with transrectal ultrasound guidance. There were 2 needle tracts on either side enabling injection at the apex, mid prostate, base, and peripheral regions (Fig. 1). Eight sites of injection were chosen with the aim of assessing drainage from all areas of the prostate and improving sensitivity.

The speed and placement of the tracer into the prostate under transrectal ultrasound guidance was improved by first placing 4 sharp guide catheters (18-gauge $250-\mathrm{mm}$ prostate seeding needle set) into position, 2 on each side, laterally and medially. Because of needle flexibility, the 18-gauge catheters were required to guide the injections. Injection from each of the 8 previously prepared syringes via a 22-gauge 150-mm Sprotte cannula (PAJUNK GmbH) was performed, in turn, under direct transrectal ultrasound vision in the sagittal plane. The 150mm-long cannulas were required to reach the base of the prostate from the perineum. It was found that deep, mid, and superficial points along the tract of the medially placed catheters would correlate with the base, central zone, and apex of the prostate. The more lateral catheter position allowed injection into the peripheral zone of the mid prostate. The 22gauge narrow cannula allowed minimal dead space in the delivery mechanism, important when delivering such a small amount of contrast. The 250-mm guide catheters were cut down to $145 \mathrm{~mm}$ to allow for the $150-\mathrm{mm}$ length of the cannula. This technique was successful in allowing rapid and controlled placement of radiotracer.

SPECT/CT Acquisition and Image Interpretation. The patient was then returned to the nuclear medicine department, where a wholebody planar exam was performed $(15 \mathrm{~cm} / \mathrm{min})$ followed by SPECT/ CT on a Symbia-6 $\gamma$-camera (Siemens Healthineers). A low-dose, unenhanced CT scan was acquired for anatomic correlation, followed by SPECT using a step-and-shoot, noncircular orbit with 64 angles (32 per head) at $15 \mathrm{~s}$ per frame. The purpose of the SPECT/CT was to familiarize all staff with the study procedures in preparation for the experimental PET/CT tracer injection. Images were acquired at 2 time points after injection: $45 \mathrm{~min}$ and $90-120 \mathrm{~min}$. SLN uptake has been reported to reach steady state after $150 \min (13)$.

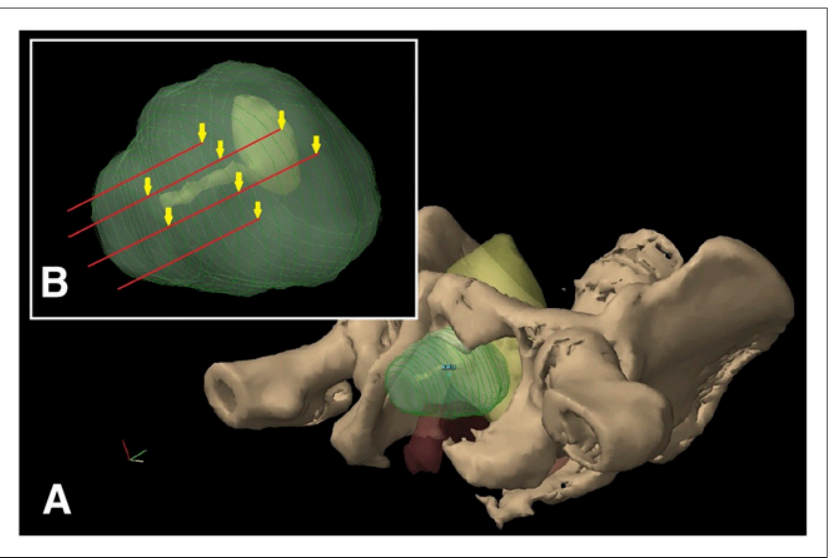

FIGURE 1. (A) Diagram of pelvis from inferolateral view. Bony-anatomy cutaway shows prostate (green, not to scale), bladder and urethra (yellow), and rectum (orange). (B) Inset shows needle insertion tracts, with arrows indicating sites of injection along these tracts.
Images were analyzed by an experienced nuclear medicine physician. SLNs were defined in accordance with the results of the consensus panel convened in 2016 (4): "All nodes that appear first in each drainage basin as seen on early (15 min) lymphoscintigrams and/or late lymphoscintigrams (and/or. . SPECT-CT imaging) in new basins that were not yet seen on the early images." Other nodes were defined as second-echelon nodes.

\section{Technique for ${ }^{68} \mathrm{Ga}-$ Nanocolloid PET/CT \\ Lymphoscintigraphy}

PET Tracer. ${ }^{68} \mathrm{Ga}$ was eluted from an onsite ${ }^{68} \mathrm{Ge} /{ }^{68} \mathrm{Ga}$ generator (iThemba; IBD Holland BV) and purified using a method previously described (15). Iron oxide nanoparticles with a 20 -nm diameter consisting of a 15-nm iron core with a coating of dextran were compounded on site using previously validated techniques (16-18). Such nanoparticles have been used as a paramagnetic contrast agent for clinical MRI. The amount of iron oxide nanoparticle administered for PET imaging is less than $1 / 100,000$ of the usual dose used for MRI. Transmission electron microscopy confirmed discrete nanoparticles with a 20 -nm size. These were radiolabeled with diethylenetriaminepentaacetic acid as the chelating agent onto the functionalized amine moiety of the dextran coat. The final product was quality-assured for radiochemical purity and sterilized via terminal filtration through a $0.22-\mu \mathrm{m}$ filter before dispensing. The product was divided into 8 sterile syringes for administration (Fig. 2).

A review of the literature provided good information for dose if using ${ }^{99 \mathrm{~m}} \mathrm{Tc}$, but the dose for a PET tracer would be much less because of the greater sensitivity of image acquisition. The dose prepared was approximately $8 \times 10 \mathrm{MBq}$ per patient. The actual dose administered was the difference between the initial and residual doses left in the syringes and needles and was less than $80 \mathrm{MBq}$ in total because of the short ${ }^{68} \mathrm{Ga}$ half-life of $68 \mathrm{~min}$. Because our study was a pilot study using interstitial injection of a PET tracer, it was unknown what the ideal dose for administration should be. On the basis of the initial imaging results, we were confident that the activity in each injection could be decreased while still allowing SLN detection. This decrease potentially extends the number of patients who could be scanned from a given production and limits exposure of operators to radiation. The mean administered activity (with SD) of the ${ }^{68} \mathrm{Ga}$-nanocolloid was $12.02 \pm 6.87 \mathrm{MBq}$ (range, 3.1-21.0 MBq) (Table 1).

PET/CT Injection Technique. Exactly the same injection technique as that trialed for SPECT/CT lymphoscintigraphy, described above, was used.

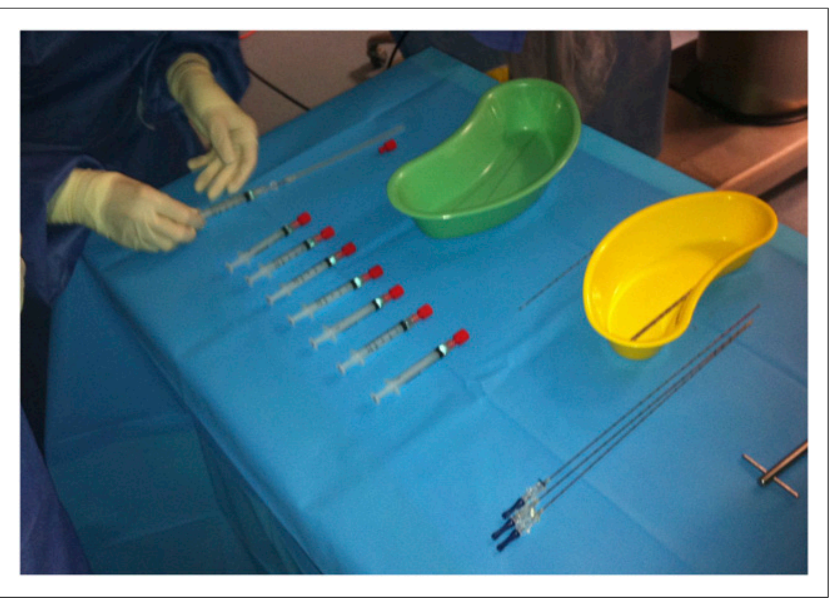

FIGURE 2. Eight syringes, each containing $0.2 \mathrm{~mL}$ of ${ }^{68} \mathrm{Ga}$-iron oxide. First syringe to be used is attached to 22-gauge 150-mm Sprotte cannula. 
TABLE 1

SLNs Identified in the Study Patients

\begin{tabular}{|c|c|c|c|c|c|c|c|}
\hline \multirow[b]{2}{*}{ Patient } & \multirow{2}{*}{$\begin{array}{l}\text { Dose }^{*} \\
(\mathrm{MBq})\end{array}$} & \multicolumn{2}{|c|}{ SLNs } & \multirow{2}{*}{$\begin{array}{l}\text { Second-echelon } \\
\text { nodes }\end{array}$} & \multicolumn{3}{|c|}{ Lymphatic drainage } \\
\hline & & Scan 1 & Scan 2 & & Classic & Aberrant & Nonlymphatic \\
\hline SPECT 1 & 82.6 & $\begin{array}{l}\text { L external iliac, } \\
\text { L common iliac }\end{array}$ & $\begin{array}{l}\text { L external iliac, L common } \\
\text { iliac }\end{array}$ & $\begin{array}{l}\text { Retroperitoneal } \\
\text { (paraaortic) }\end{array}$ & Yes & No & No \\
\hline SPECT 2 & 31.0 & R internal iliac & $\begin{array}{l}\text { R internal iliac, L internal } \\
\quad \text { iliac (probable) }\end{array}$ & & Yes & No & No \\
\hline SPECT 3 & 19.9 & $\mathrm{R}$ external iliac & R external iliac & $\mathrm{R}$ common iliac & Yes & No & No \\
\hline PET 1 & 16.1 & $\begin{array}{l}\text { R internal iliac, } \\
\text { L perivesical, } \\
\text { L internal iliac, } \\
\text { L external iliac (probable) }\end{array}$ & $\begin{array}{l}R \text { internal iliac, } \\
\mathbf{L} \text { perivesical }\end{array}$ & & Yes & Yes & No \\
\hline PET 2 & 8.7 & $\begin{array}{l}\mathrm{L} \text { internal iliac, } \mathbf{L} \text { anterior } \\
\text { periprostatic into } \mathbf{L} \\
\text { inferior pubic ramus }\end{array}$ & $\begin{array}{l}\mathrm{L} \text { internal iliac, } \mathbf{L} \text { anterior } \\
\text { periprostatic into } \mathbf{L} \\
\text { inferior pubic ramus }\end{array}$ & $\mathrm{L}$ internal iliac 2 & Yes & No & Yes \\
\hline PET 3 & 21.0 & $\mathrm{~L}$ periprostatic & $\begin{array}{l}\text { L periprostatic, } \mathrm{R} \\
\text { periprostatic }\end{array}$ & & Yes & No & No \\
\hline PET 4 & 11.2 & $\begin{array}{l}\text { R mesorectal, L external } \\
\text { iliac }\end{array}$ & $\begin{array}{l}\text { R mesorectal, L external } \\
\text { iliac }\end{array}$ & R presacral & Yes & Yes & No \\
\hline PET 5 & 3.1 & L internal iliac, $\mathbf{L}$ inguinal & $\begin{array}{l}\text { L internal iliac (disappears), } \\
\text { L inguinal }\end{array}$ & $\begin{array}{l}\text { L common iliac, } \\
\text { distal thoracic } \\
\text { duct (Virchow } \\
\text { node) }\end{array}$ & Yes & Yes & No \\
\hline
\end{tabular}

*Injected dose of ${ }^{99 m}$ Tc-radiocolloid for SPECT and ${ }^{68} \mathrm{Ga}$-nanocolloid for PET.

SLNs were found both in classic sites of regional spread and in aberrant sites (boldface font). First 3 patients underwent 99mTc-radiocolloid SPECT/CT, and next 5 underwent ${ }^{68} \mathrm{Ga}-$ nanocolloid PET/CT. Scans were obtained about 45 and 100 min after tracer injection.

PET/CT Acquisition and Interpretation. PET/CT scans were acquired on a Discovery STE scanner (GE Healthcare). The first scan was performed after extubation and discharge from the theater recovery unit approximately 30-40 min after intraprostatic injection. A dedicated acquisition of the pelvis was followed by imaging from the upper thighs to the neck. Low-dose CT was used for attenuation correction and anatomic localization. PET/CT imaging of the lower neck, chest, abdomen, and pelvis was repeated at 2 further time points up to $130 \mathrm{~min}$.

The images were analyzed by an experienced nuclear medicine physician. As in the SPECT/CT imaging, SLNs were defined as all nodes that appear first in each drainage basin as seen on early or late lymphoscintigrams in accordance with consensus guidelines (4). Other PET-avid nodes were defined as second-echelon nodes. The intensity of tracer distribution was measured in units of SUV and ratios of avidity as compared with other compartments and the background.

\section{Statistical Analysis}

Efficacy was defined as the capacity of the technique to identify SLNs, because such a capacity would be an indicator of successful lymphoscintigraphy technique. We aimed to demonstrate that at least 1 sentinel node could be identified in at least two thirds of cases with greater than $80 \%$ confidence. The study was monitored using a Bayesian trial design based on a conservative (noninformative) uniform prior (19). A minimum of 5 and maximum of 20 cases were to be enrolled for the ${ }^{68} \mathrm{Ga}$ component of the study. Any adverse reactions during or after administration of the radiotracer were recorded. Once 5 cases had been enrolled, event rates were continuously monitored for feasibility and futility. If no negative scans were seen in the first 5 cases, the estimated SLN identification rate would be $86 \%$, with a $91 \%$ probability that SLNs would be identified in more than $66 \%$ of cases. The technique could then be considered adequate and consideration given to ceasing further accrual.

\section{RESULTS}

Eight men, aged 66-83 y (mean age, 73 y), participated in the study between December 2013 and February 2015. The first three underwent SPECT/CT lymphoscintigraphy, and the last five underwent PET/CT. There were no adverse or clinically detectable pharmacologic effects. No significant changes in vital signs were observed.

Aberrant SLNs were found in 3 of the 5 participants (perivesicular, mesorectal, and inguinal lymph nodes) who underwent PET/CT imaging. Furthermore, in 1 participant a second-echelon distal left thoracic duct region (Virchow node) was visualized in the absence of upper abdominal or other supradiaphragmatic uptake (Fig. 3). Second-echelon mesorectal and retroperitoneal nodes were also noted. In one patient, a curvilinear drainage pathway extending from the prostate bed to the pubic ramus was observed. We postulate that this observation reflected a venous plexus rather than a lymphatic pathway (Fig. 4).

SLNs were successfully identified in all $5{ }^{68}$ Ga-nanocolloid PET/CT cases, sufficient to satisfy the feasibility objective. ${ }^{68} \mathrm{Ga}$ 


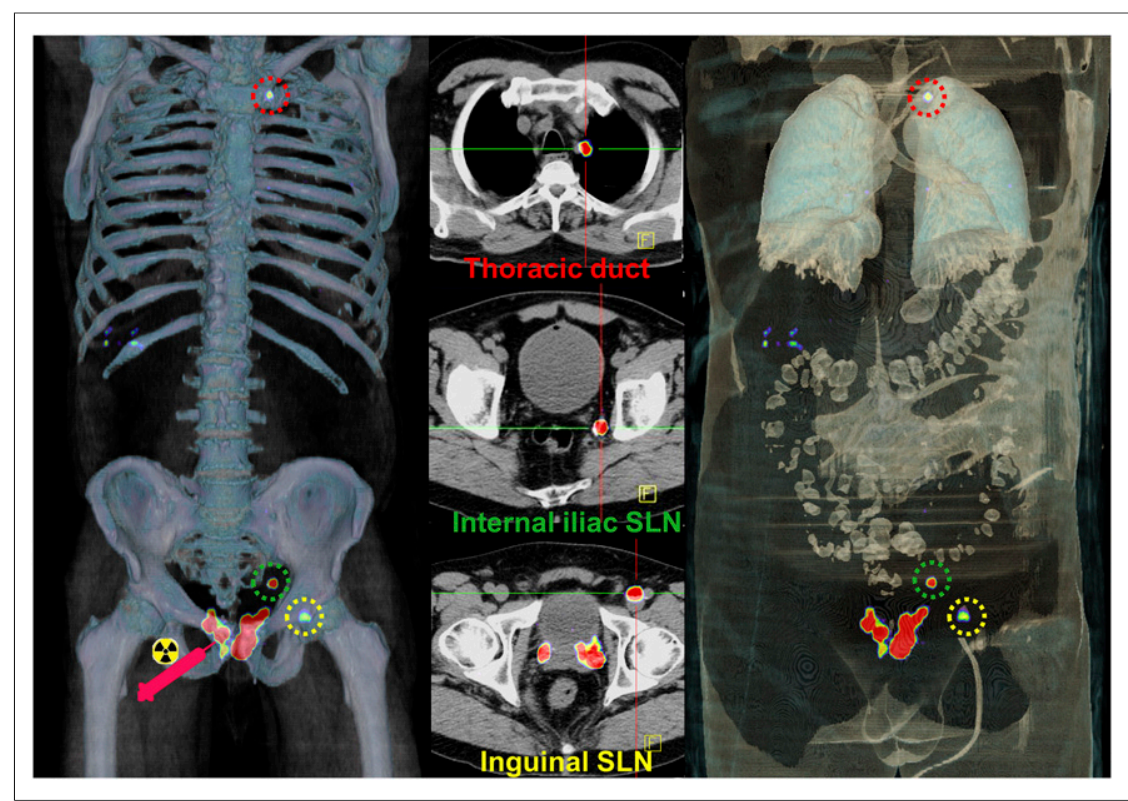

FIGURE 3. Volume-rendered PET/CT (left and right) images and axial PET/CT (middle) images. After injection of tracer into prostate, imaging at 45 min demonstrated internal iliac SLN, inguinal SLN, and focal uptake at terminal thoracic duct near entry into left subclavian vein. No intervening SLN stations were seen in abdomen or chest. This intriguing finding correlates with increasing recognition of Virchow nodal metastases visualized with ${ }^{68} \mathrm{Ga}-\mathrm{PSMA}$ or ${ }^{18} \mathrm{~F}$-choline PET.

avidity was still visible in SLNs at the second time point, approximately $85-120 \mathrm{~min}$ after commencement of intraprostatic tracer injection. One site initially had high uptake but did not retain ${ }^{68} \mathrm{Ga}$ avidity at the later time point-a finding that we interpreted as an area of lymphatic pooling rather than a sentinel node. Table 1 lists the SLN sites identified. In one patient, contrast was able to reflux into the bladder neck, indicating passage from the interstitial space into the prostatic urethra.

\section{DISCUSSION}

PET/CT lymphoscintigraphy with a novel ${ }^{68} \mathrm{Ga}$-iron oxide nanocolloid was successful in all our patients. As well as easily identifying the site of SLNs, the technique also identified secondary echelons of distant lymphatic spread. Aberrant lymphatic pathways, similar to those reported in the SPECT imaging literature, were noted in 3 of 5 participants $(3,10)$. Spread of tracer to periprostatic, mesorectal, and perivesical sites was seen. In one patient, tracer could be seen directly tracking to the bony pubic ramus, consistent with an anatomic basis for the preferential spread of prostate cancer to bones as hypothesized by Batson (20). He proposed a valveless connection between the prostatic venous plexus and the vertebral venous plexus and demonstrated that dye in the prostatic plexus would preferentially fill the vertebral veins rather than the caval system if intraabdominal pressure was raised to simulate that of coughing, straining, or lifting. This hypothesis is supported by the higher incidence of bone metastases in the pelvis and lower vertebrae in patients with prostate cancer than in patients with breast or lung cancer $(6,21,22)$. This tendency for spread to the lumbar and pelvic bones is seen more obviously when the patient has three or fewer metastases, suggesting that this site is preferred in the early stage of metastasis, hypothetically before the cells reach the lungs and the arterial side of the circulation (6).
The distribution of SLNs was similar to that previously reported, confirming the potential for lymphatic spread in an unpredictable pattern, outside the classic standard and extended pelvic lymph node dissection templates. We hypothesize that failure to manage this unpredictability with SLN imaging has led to the lack of success with standard treatment templates for standard or extended pelvic lymph node dissection and for whole-pelvis radiation, with a significant proportion of high-risk sites for disease being missed. SLN biopsy in prostate cancer is still considered experimental, and its role in improving oncologic outcome remains controversial $(4,23)$. We propose a further study to obtain a more detailed picture of anatomic variation and to demonstrate whether lymphaticovenous connection to adjacent bone is a common anatomic finding. The use of whole-body PET imaging in this study enabled visualization of a distal left thoracic duct region (Virchow node), which is also a documented pattern of spread (24).

Knowledge of the site of sentinel node anatomic distribution through correlation with PET/CT anatomy allows planning of nodal sampling, although it is still potentially subject to sampling error. Coadministration of a ${ }^{99 \mathrm{~m}} \mathrm{Tc}$ or fluorescent tracer could guide intraoperative localization of sentinel nodes. The availability of intraoperative probes suitable for detection of positron-emitting radionuclides is also advancing (25). In the future, it would be interesting to use a long-lived PET tracer, such as ${ }^{64} \mathrm{Cu}$ or ${ }^{89} \mathrm{Zr}$.

We use multiparametric MRI and ${ }^{68}$ Ga-PSMA-11 in most highrisk cases to identify sites of macroscopic regional nodal involvement and to exclude distant disease. In such cases, treatment is planned in the context of positive findings. However, whereas PSMA is both quite sensitive and specific for nodal involvement, it is clear that microscopic foci can be missed (26). Our PET/CT lymphoscintigraphy technique could be used as an adjunct to definitive surgery (after ${ }^{68} \mathrm{Ga}$-PSMA staging) for those men with a high risk of microscopic nodal disease.

A weakness of our study was that we did not compare sensitivity and specificity of the PET tracer against a ${ }^{99 \mathrm{~m}} \mathrm{Tc}$-nanocolloid, a fluorescent tracer, or a hybrid of these. The initial study using ${ }^{99 \mathrm{~m}} \mathrm{Tc}$ allowed us to validate our injection technique without the time pressure associated with ${ }^{68} \mathrm{Ga}$, the short half-life of which necessitates fairly rapid injection and imaging. We aimed to test the feasibility of such a logistically complex process before moving to an experimental arm. Because the study was not used for clinical treatment planning, there were ethical constraints on subjecting the patient to both PET/CT and SPECT/CT imaging. In the future, such would be feasible with coinjection of both tracers to confirm concordance of sentinel node identification.

The technique we described is probably not practical for clinical use because we perform it under general anesthetic and the 68-min half-life of the gallium tracer means the PET scanner needs to be available for use at a prescribed 30-100 min after injectiona difficult requirement in a busy nuclear medicine department. 


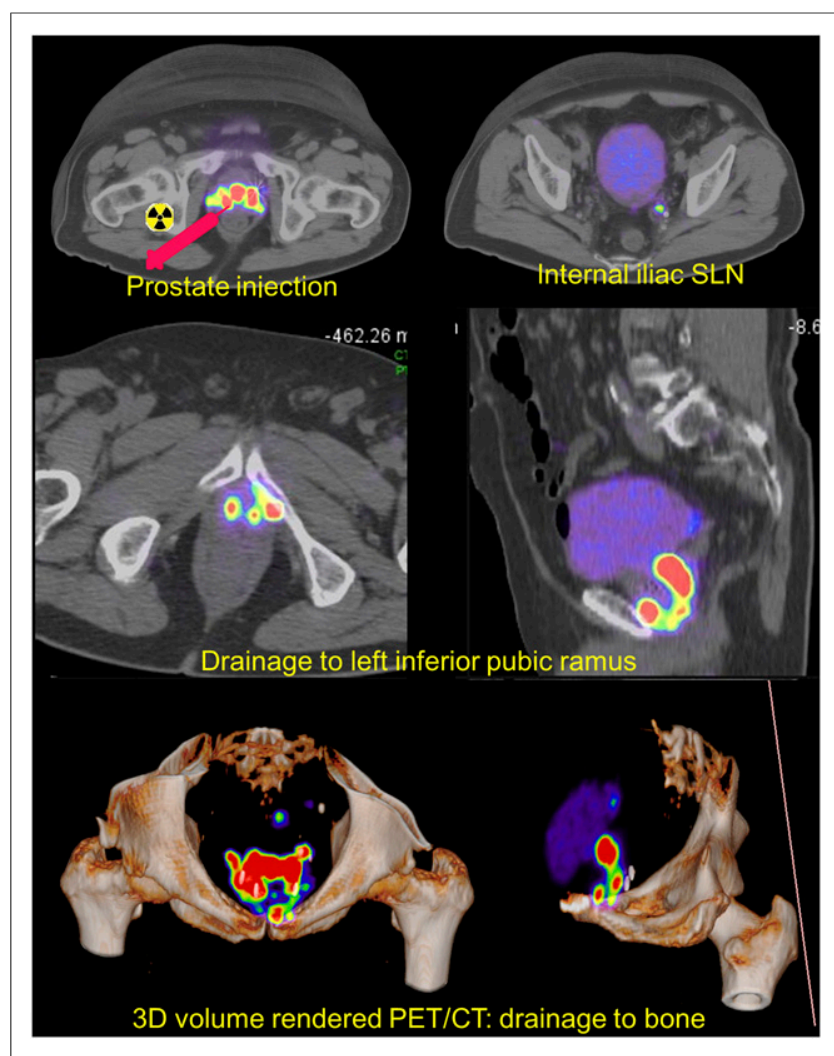

FIGURE 4. After injection in prostate, left internal iliac SLN was identified. Additional drainage pathway from periprostatic region to inferior pubic ramus was observed and could represent drainage via periprostatic venous plexus rather than lymphatic pathway. Finding shows locoregional rather than hematogenous pathway of metastasis to pelvic bones and explains predominance of pelvic bony metastases in some patients.

Investigators were required to wheel the patient from the recovery room directly to the PET scanner as soon as the patient had adequately awakened from the anesthetic. The technique could be adapted for transrectal injection to only 4 sites per patient, which is thought to be optimal for SLN assessment in clinical practice (4). For this anatomic study, we wanted to conscientiously ensure that drainage from all regions of the prostate was accounted for.

The use of ${ }^{68} \mathrm{Ga}$ is timely in the context of rising interest in ${ }^{68} \mathrm{Ga}$-PSMA PET/CT (27). Like the ${ }^{99 \mathrm{~m} T c}$ used for SPECT/CT imaging, ${ }^{68} \mathrm{Ga}$ is a generator-produced radioisotope, obviating the cyclotron required for ${ }^{18} \mathrm{~F}$ PET imaging. ${ }^{68} \mathrm{Ga}$ generators have a shelf-life of 6-12 mo, compared with $2 \mathrm{wk}$ for a ${ }^{99 \mathrm{~m}} \mathrm{Tc}$ generator. For centers performing ${ }^{68} \mathrm{Ga}$-PSMA PET/CT, there is potential to use the generator for sentinel node imaging as well. Interest in ${ }^{68} \mathrm{Ga}$ radiotracers is rapidly growing because of the superior temporal and spatial resolution of PET compared with conventional SPECT imaging (7). In addition, the ${ }^{68} \mathrm{Ga}$ radiation dose to the patient is similar to the radiation dose of conventional imaging because of its short half-life of $68 \mathrm{~min}$. The use of an iron oxide nanoparticle potentially also allows MRI, although this would require a substantially higher particulate mass.

For lymphoscintigraphy, there are several potential advantages of PET over SPECT. One is the ability to perform 3-dimensional dynamic imaging. Although dynamic imaging is possible with conventional nuclear medicine, it is limited to 2-dimensional planar imaging. The slow speed of SPECT/CT means it is limited to static rather than dynamic imaging. PET also enables rapid whole-body 3D imaging, which in this small series enabled identification of a Virchow second-echelon node. To date, lymphoscintigraphy imaging is limited to simply showing potential pathways for metastatic spread rather than actual tumor spread.

\section{CONCLUSION}

Lymphoscintigraphy using a novel ${ }^{68} \mathrm{Ga}$-nanocolloid PET tracer was successfully performed without observed side effects. In 3 of 5 participants, unexpected potential pathways for transit of malignant cells were discovered, with rapid tracer drainage being identified to pelvic bone and to perivesicular, mesorectal, inguinal, and Virchow nodes. The prevalence of both aberrant and nonlymphatic pathways of spread could be further investigated with this technique.

\section{DISCLOSURE}

This work was supported by a grant from the Peter MacCallum Foundation (2012), by a Faculty of Radiation Oncology Genitourinary Group (FROGG) fellowship grant (2013) under the auspices of the Royal Australia and New Zealand College of Radiology (RANZCR), and by an Australian Government Research Training Program (RTP) scholarship. No other potential conflict of interest relevant to this article was reported.

\section{ACKNOWLEDGMENT}

We thank Dr. Giovanni Mandarano for his generous provision of the iron oxide nanocolloid for this study, developed during his time at the School of Medical Sciences, RMIT University, Australia.

\section{REFERENCES}

1. Incidence - SEER 18 Regs Research Data + Hurricane Katrina Impacted Louisiana Cases, Nov 2012 Sub (1973-2010 varying). SEER*Stat databases: November 2012 submission. Surveillance, Epidemiology, and End Results Program website. https://seer.cancer.gov/data-software/documentation/seerstat/nov2012/. Accessed September 4, 2018.

2. SEER*Stat software. Surveillance, Epidemiology, and End Results Program website. https://seer.cancer.gov/seerstat/. Released April 15, 2013. Accessed September 4, 2018.

3. Vermeeren L, Meinhardt W, van der Poel HG, Valdes Olmos RA. Lymphatic drainage from the treated versus untreated prostate: feasibility of sentinel node biopsy in recurrent cancer. Eur J Nucl Med Mol Imaging. 2010;37: 2021-2026.

4. van der Poel HG, Wit EM, Acar C, et al. Sentinel node biopsy for prostate cancer: report from a consensus panel meeting. BJU Int. 2017;120: 204-211.

5. Gandaglia G, Abdollah F, Schiffmann J, et al. Distribution of metastatic sites in patients with prostate cancer: a population-based analysis. Prostate. 2014;74: 210-216.

6. Styles C. The distribution of bone metastases as shown on isotope scanning: proposed modes of spread. Australas Radiol. 1989;33:226-228.

7. Hicks RJ, Hofman MS. Is there still a role for SPECT-CT in oncology in the PET-CT era? Nat Rev Clin Oncol. 2012;9:712-720.

8. Ganswindt U, Paulsen F, Corvin S, et al. Intensity modulated radiotherapy for high risk prostate cancer based on sentinel node SPECT imaging for target volume definition. BMC Cancer. 2005;5:91.

9. Wawroschek F, Vogt H, Wengenmair H, et al. Prostate lymphoscintigraphy and radio-guided surgery for sentinel lymph node identification in prostate cancer: technique and results of the first 350 cases. Urol Int. 2003;70:303-310.

10. Vermeeren L, Valdes Olmos RA, Meinhardt W, et al. Value of SPECT/CT for detection and anatomic localization of sentinel lymph nodes before laparoscopic sentinel node lymphadenectomy in prostate carcinoma. J Nucl Med. 2009;50: $865-870$. 
11. Stroup SP, Kane CJ, Farchshchi-Heydari S, et al. Preoperative sentinel lymph node mapping of the prostate using PET/CT fusion imaging and Ga-68-labeled tilmanocept in an animal model. Clin Exp Metastasis. 2012;29:673-680.

12. Buckle T, Brouwer OR, Valdes Olmos RA, van der Poel HG, van Leeuwen FW. Relationship between intraprostatic tracer deposits and sentinel lymph node mapping in prostate cancer patients. J Nucl Med. 2012;53:1026-1033.

13. Wawroschek F, Wengenmair H, Senekowitsch-Schmidtke R, et al. Prostate lymphoscintigraphy for sentinel lymph node identification in canines: reproducibility, uptake, and biokinetics depending on different injection strategies. Urol Res. 2003;31:152-158

14. Mattei A, Fuechsel FG, Bhatta Dhar N, et al. The template of the primary lymphatic landing sites of the prostate should be revisited: results of a multimodality mapping study. Eur Urol. 2008;53:118-125.

15. Zhernosekov KP, Filosofov DV, Baum RP, et al. Processing of generator-produced ${ }^{68} \mathrm{Ga}$ for medical application. J Nucl Med. 2007;48:1741-1748.

16. Park J, An K, Hwang Y, et al. Ultra-large-scale syntheses of monodisperse nanocrystals. Nat Mater. 2004;3:891-895.

17. Lin MM, Kim do K, El Haj AJ, Dobson J. Development of superparamagnetic iron oxide nanoparticles (SPIONS) for translation to clinical applications. IEEE Trans Nanobioscience. 2008;7:298-305.

18. Hradil J, Pisarey A, Babic M, Horal D. Dextran-modified iron oxide nanoparticles. China Particuology. 2007;5:162-168.
19. Mehta CR, Cain KC. Charts for the early stopping of pilot studies. J Clin Oncol. 1984;2:676-682.

20. Batson OV. The function of the vertebral veins and their role in the spread of metastases. Ann Surg. 1940;112:138-149.

21. Cumming J, Hacking N, Fairhurst J, Ackery D, Jenkins JD. Distribution of bony metastases in prostatic carcinoma. Br J Urol. 1990;66:411-414.

22. Bubendorf L, Schopfer A, Wagner U, et al. Metastatic patterns of prostate cancer: an autopsy study of 1,589 patients. Hum Pathol. 2000;31:578-583.

23. Wit EMK, Acar C, Grivas N, et al. Sentinel node procedure in prostate cancer: a systematic review to assess diagnostic accuracy. Eur Urol. 2017;71:596605.

24. Jones H, Anthony PP. Metastatic prostatic carcinoma presenting as left-sided cervical lymphadenopathy: a series of 11 cases. Histopathology. 1992;21:149154.

25. González SJ, Gonzalez L, Wong J, et al. An analysis of the utility of handheld PET probes for the intraoperative localization of malignant tissue. $J$ Gastrointest Surg. 2011;15:358-366.

26. Jilg CA, Drendel V, Rischke HC, et al. Diagnostic Accuracy of Ga-68-HBEDCC-PSMA-ligand-PET/CT before salvage lymph node dissection for recurrent prostate cancer. Theranostics. 2017;7:1770-1780.

27. Hofman MS, Iravani A. Gallium-68 prostate-specific membrane antigen PET imaging. PET Clin. 2017;12:219-234. 